\title{
Perfil clínico de pacientes com diagnóstico de tuberculose atendidos no Hospital Universitário de Londrina, Paraná
}

\section{Clinical profile of patients diagnosed with tuberculosis treated at the University Hospital of Londrina, Paraná}

\author{
Larissa Rodrigues Bosqui ${ }^{1}$, Suelen Santos Silva ${ }^{2}$, Raquel Arruda Sanfelice ${ }^{3}$, Milena Mengazzo \\ Miranda-Sapla ${ }^{1}$, Daniele Sapede Alvarenga ${ }^{1}$, Bruno Bevenuto Lucas ${ }^{4}$, Laís Fernanda Machado ${ }^{5}$, \\ Francine Nesello Melanda ${ }^{6}$, Luiz Antonio Custodio ${ }^{7}$, Wander Rogerio Pavanelli ${ }^{8}$, Ivete Conchon- \\ Costa $^{9}$, Idessania Nazareth Costa ${ }^{10}$
}

\begin{abstract}
Resumo
O objetivo deste estudo consistiu em analisar o perfil clínico de pacientes positivos para tuberculose através de um estudo epidemiológico, descritivo e retrospectivo, com base em dados secundários contidos em prontuários dos pacientes atendidos no Hospital Universitário de Londrina compreendendo de janeiro de 2010 a dezembro de 2014. Dos prontuários disponíveis para a análise no momento de estudo 86 casos eram positivos para tuberculose, sendo a maioria do sexo masculino $(65 / 76 \%)$ com faixa etária compreendida entre 2 e 91 anos. Os principais sinais clínicos apresentados foram tosse $(50 / 58 \%)$, febre $(45 / 52 \%)$ e perda de peso $(34 / 40 \%)$. Em relação à forma clinica, $58 / 67 \%$ dos pacientes apresentaram a forma pulmonar, e $28 / 33 \%$ a forma não pulmonar. Casos positivos para tuberculose em associação com HIV/AIDS corresponderam a 32/37\%. Também foram relatados hábitos prejudiciais dos pacientes nos quais 30/34\% eram tabagistas, $20 / 23 \%$ usuários de drogas e 14/16\% etilistas. Diante da escassez de dados publicados referentes à tuberculose na nossa região e sua relevância para a saúde pública, nosso estudo contribui com os aspectos epidemiológicos principalmente em relação ao elevado número de casos de coinfecção com Vírus da Imunodeficiência Humana e pacientes com evolução a óbito, auxiliando assim, o desenvolvimento e implementação de campanhas ou projetos que visem o diagnóstico e tratamento precoce.
\end{abstract}

Palavras chave: Tuberculose. Saúde pública. Epidemiologia.

\footnotetext{
${ }^{1}$ Doutoranda em Patologia Experimental pela Universidade Estadual de Londrina. Laboratório de Parasitologia Experimental, Departamento de Ciências Patológicas, Universidade Estadual de Londrina; Londrina, Paraná, Brasil.

${ }^{2}$ Doutorado em Patologia Experimental pela Universidade Estadual de Londrina. Laboratório de Parasitologia Experimental, Departamento de Ciências Patológicas, Universidade Estadual de Londrina; Londrina, Paraná, Brasil.

${ }^{3}$ Mestrado em Patologia Experimental pela Universidade Estadual de Londrina. Laboratório de Parasitologia Experimental, Departamento de Ciências Patológicas, Universidade Estadual de Londrina; Londrina, Paraná, Brasil.

${ }^{4}$ Graduando em Odontologia pela Universidade Estadual de Londrina. Laboratório de Parasitologia Experimental, Departamento de Ciências Patológicas, Universidade Estadual de Londrina; Londrina, Paraná, Brasil.

${ }^{5}$ Graduanda em Ciências Biológicas pela Universidade Estadual de Londrina. Laboratório de Parasitologia Experimental, Departamento de Ciências Patológicas, Universidade Estadual de Londrina; Londrina, Paraná, Brasil.

${ }^{6}$ Doutoranda em Saúde Coletiva pela Universidade Estadual de Londrina. Laboratório de Parasitologia Experimental, Departamento de Ciências Patológicas, Universidade Estadual de Londrina; Londrina, Paraná, Brasil.

${ }^{7}$ Doutorado em Patologia Experimental pela Universidade Estadual de Londrina. Ambulatório de Especialidades do Hospital Universitário de Londrina, Paraná, Brasil. ${ }^{8}$ Doutorado em Imunologia Básica e Aplicada pela Universidade de São Paulo. Laboratório de Parasitologia Experimental, Departamento de Ciências Patológicas, Universidade Estadual de Londrina; Londrina, Paraná, Brasil.

${ }^{9}$ Doutorado em Microbiologia pela Universidade Estadual de Londrina. Laboratório de Parasitologia Experimental, Departamento de Ciências Patológicas, Universidade Estadual de Londrina; Londrina, Paraná, Brasil.

${ }^{10}$ Doutorado em Imunologia e Parasitologia Aplicadas pela Universidade Federal de Uberlândia. Laboratório de Parasitologia Experimental, Departamento de Ciências Patológicas, Universidade Estadual de Londrina; Londrina, Paraná, Brasil.
} 


\begin{abstract}
The objective of this study was to analyze the clinical profile of patients positive for tuberculosis through an epidemiological study, descriptive and retrospective, based on secondary data contained in medical records of patients that attended the Hospital Universitário de Londrina comprising from January 2010 to December 2014. From the medical records available for analysis at the moment of study 86 cases were positive for tuberculosis, being the majority male $(65 / 76 \%)$ with age group comprised between 2 to 91 years. The main symptoms presented were cough (50/58\%), fever $(45 / 52 \%)$ and weight loss (34/40\%). Regarding the clinical form, 58/67\% of the patients presented the pulmonary form, and $28 / 33 \%$ the non-pulmonary form. Positive cases for tuberculosis in association with HIV/AIDS corresponded to $32 / 37 \%$. Harmful habits were also reported in patients in which $30 / 34 \%$ were smokers, $20 / 23 \%$ drug users and 14/16\% were alcoholics. Against scarcity of published data referring to tuberculosis in our region and its relevance to public health, our study contributes to the epidemiological aspects mainly in relation to the high number of cases of coinfection with Human Immunodeficiency Virus and patients with evolution to death, assisting the development and implementation of campaigns or projects aimed at early diagnosis and treatment.
\end{abstract}

Keyword: Tuberculosis. Public health. Epidemiology.

\section{Introdução}

A tuberculose (TB) é uma doença infectocontagiosa causada pela bactéria intracelular Mycobacterium tuberculosis ou bacilo de Koch (BK), que afeta prioritariamente os pulmões, embora possa acometer outros órgãos e sistemas (WHO, 2015).

A transmissão da TB é direta e aérea, quase exclusivamente por aerossóis de tosse e a aglomeração de pessoas apresenta-se como o principal fator de transmissão. Má alimentação, falta de higiene, tabagismo, alcoolismo ou qualquer outro fator que gere baixa resistência orgânica, também favorece o estabelecimento da TB (SHETE et al., 2015).

A exposição à $M$. tuberculosis na maioria dos casos é assintomática, podendo causar em alguns pacientes sintomas semelhantes ao de uma gripe. Tosse seca e contínua, posteriormente com secreção e com duração de mais de quatro semanas, sudorese noturna, cansaço excessivo, palidez, falta de apetite e rouquidão são os sintomas da doença. Dificuldade na respiração, eliminação de sangue e acúmulo de pus na pleura pulmonar são característicos em casos mais graves (BRASIL, 2015; SHETE et al., 2015).

A TB é capaz de comprometer mais de um órgão em um mesmo paciente, podendo expressar-se por formas disseminadas como a cutânea aguda disseminada (miliar) ou extrapulmonares. As formas clínicas extrapulmonares são decorrentes da disseminação dos bacilos pela corrente sanguínea e/ou linfática, a partir do foco de inoculação inicial no pulmão, e são classificadas segundo a localização: pleural, ganglionar periférica, osteoarticular, geniturinária e meningoencefálica (ARORA et al., 2016; SANDGREN; HOLLO; VAN DER WERF, 2013).

Todas essas formas clínicas estão intimamente relacionadas com a resposta imune. A resposta imune tem papel fundamental na defesa contra $M$. tuberculosis, constituindo o principal impedimento para a ocorrência das infecções disseminadas que estão associadas ao elevado índice de mortalidade (O'GARRA et al., 2013). No entanto, a resposta imune contra $M$. tuberculosis é complexa e incompletamente caracterizada, o que dificulta o desenvolvimento de novos testes, vacinas e tratamentos.

Embora seja evidente o papel fundamental das células T CD4 ${ }^{+}$juntamente com as citocinas interleucina 12 (IL-12), interferon gama (IFN- $\gamma$ ) e fator de necrose tumoral alfa (TNF- $\alpha)$ no controle da infecção por M. tuberculosis, ainda não há compreensão exata dos fatores que determinam a resistência de alguns indivíduos frente à infecção por M. tuberculosis, enquanto outros são susceptíveis (BINI et al., 2015; CLIFFORD et al., 2015; ETNA et al., 2014; GEORGE et al., 2015; HOSSAIN; NORAZMI, 2013; KASSA et al., 2016; LAI et al., 2013; WU et al., 2017). Entretanto, sabe-se que a imunidade protetora de $M$. tuberculosis pode impedir a multiplicação do bacilo, sem a eliminação completa deste patógeno.

Dados epidemiológicos demonstram que um terço da população mundial está infectada com $M$. tuberculosis e segundo a Organização Mundial de Saúde aproximadamente nove milhões de novos casos de TB foram relatados em 2008 (WHO, 2015). 
Atualmente, o Brasil ocupa a $16^{\mathrm{a}}$ posição entre os 22 países com maior carga de TB e a $111^{\mathrm{a}}$ posição em nível de incidência (WHO, 2015). Desta forma, a tuberculose continua sendo a segunda principal causa de morte de doença infecciosa em todo o mundo (AMARE et al., 2015). Segundo Brasil (2014), o Brasil registrou 71.230 novos casos de TB em 2013. A incidência da doença no país foi de 36,7 casos para cada 100 mil habitantes, o que indica queda de $20,3 \%$ em relação a 2003 , quando a taxa era de 44,4 casos para cada 100 mil pessoas.

No período de 2015 a 2016 houve 3.044 casos de tuberculose confirmados no estado do Paraná. Destes casos, 282 foram notificados na região de Londrina. Neste mesmo período o município de Londrina apresentou $75 \%$ dos casos notificados como forma pulmonar e $15 \%$ dos casos TB notificados apresentavam coinfecção com o Vírus da Imunodeficiência Humana (HIV) (BRASIL, 2016).

Embora a TB seja uma das maiores causas de mortalidade e morbidade em muitos países, e um problema de saúde de alta relevância, o sucesso no tratamento desta doença é largamente dependente da adesão do paciente ao esquema adotado (BOGAZ, 2016). No Brasil, o esquema terapêutico atual é altamente efetivo com capacidade para curar praticamente todos os casos sendo fornecido gratuitamente pela Rede Pública, através do Programa Nacional de Controle da Tuberculose (BRASIL, 2015).

Em 1993, a OMS declarou a TB uma emergência mundial, utilizando a estratégia do Tratamento Diretamente Observado (Directly Observed Treatment, Short-course - DOTS) para atingir um maior percentual de cura e de detecção dos casos e reduzir o abandono ao tratamento (WHO, 2015).

Esta estratégia foi elaborada com base em cinco elementos fundamentais: detecção dos casos de TB por baciloscopia entre pacientes sintomáticos respiratórios que demandam os serviços gerais de saúde; tratamento padronizado de curta duração, diretamente observável e monitorado em sua evolução; provisão regular de medicamentos; utilização de sistema de registro e informação que assegure a avaliação do tratamento e o compromisso político colocando o controle da TB como prioridade entre as políticas de saúde (EL KAMEL et al., 2015).

Em 1979, o sistema de tratamento preconizado para TB era composto pelo esquema I Rifampicina + Isoniazida + Pirazinamida (RHZ), no entanto, a partir de 2009, foi introduzido o etambutol como quarto fármaco na fase intensiva de tratamento, devido ao aumento da resistência primária à isoniazida. $\mathrm{O}$ esquema básico (RHZE) é indicado para casos novos de tuberculose pulmonar e extrapulmonar (exceto a forma meningoencefálica) de adultos e adolescentes. A apresentação farmacológica desse esquema passa a ser em comprimidos de doses fixas combinadas dos quatro medicamentos (RHZE), nas seguintes dosagens: R 150mg, H 75mg, Z 400mg e E $275 \mathrm{mg}$. Nos casos de recidiva após cura ou retorno após abandono de tratamento, inicia-se o esquema básico, mas é solicitada cultura de escarro e teste de sensibilidade para escolha dos fármacos (BRASIL, 2015).

Nos casos de pacientes que apresentem hepatopatias, efeitos colaterais ao esquema RHZE, ou que fazem uso de imunossupressores ou ainda pacientes com HIV ou Síndrome da Imunodeficiência Adquirida (AIDS), tuberculose meningoencefálica ou que apresentem resistência ao tratamento RHZE, o esquema padronizado é para multirresistência ou esquemas especiais individualizados (BRASIL, 2015).

Diante do aumento da incidência de TB, tanto em regiões tropicais quanto subtropicais, se faz necessário a realização de métodos de ações e buscas envolvendo governos, membros das Nações Unidas e órgãos de comunidade científica que trabalhem arduamente para que a mortalidade referente tanto à tuberculose, quanto à outras infecções associadas ou não a essa doença, como por exemplo, HIV/AIDS, sejam minimizadas (PONCE et al., 2016).

Em virtude da relevância da tuberculose para a saúde pública e diante da escassez de dados publicados referentes ao assunto na nossa região, o nosso objetivo foi analisar o perfil clínico de pacientes positivos para tuberculose e que foram atendidos no Hospital Universitário de Londrina no período de 2010 a 2014.

\section{Material e Método}

Trata-se de um estudo epidemiológico, descritivo, retrospectivo com base de dados secundários contidos em prontuários dos pacientes. A pesquisa foi realizada no período de janeiro de 2010 a dezembro de 2014, por meio da análise de prontuários de pacientes portadores de tuberculose atendidos no Hospital Universitário de Londrina-PR. Todos os pacientes foram identificados através da busca eletrônica ao sistema do hospital, e incluídos no estudo. Em seguida, os prontuários correspondentes aos números identificados foram localizados e procedeu-se as coletas de dados dos prontuários de pacientes diagnosticados com TB, 
principalmente por baciloscopia, cultura e biópsia. No período de janeiro de 2010 a dezembro de 2014 foram registrados eletronicamente 94 casos positivos para tuberculose, sendo 86 prontuários ativos. Outras informações coletadas foram referentes a sexo, idade, aspectos clínicos, tipo de tuberculose diagnosticada (tuberculose pulmonar e não pulmonar), hábitos como tabagismo, uso de álcool e drogas ilícitas, associação com HIV/AIDS, esquema de tratamento e casos de óbitos. Os dados foram catalogados em planilha do programa Excel, e a análise descritiva e representações gráficas foram realizadas por meio de frequências absolutas e relativas utilizando o programa GraphPad Prism 5.

Este estudo foi aprovado pelo Comitê de Ética em Pesquisa em Seres Humanos da Universidade Estadual de Londrina, n. 1.306.715.

\section{Resultados}

Dentre os 86 prontuários ativos para acesso no momento da coleta de dados, a frequência observada foi maior para o sexo masculino (65/76\%). A faixa etária dos pacientes avaliados variou entre 2 e 91 anos com média de 41,7 e desvio padrão 17,3.

Dentre os sinais clínicos apresentados, os mais frequentes foram tosse $(50 / 58 \%)$, febre $(45 / 52 \%)$ e perda de peso (34/40\%) (Tabela 1).

Em relação ao tipo de TB diagnosticada 58/67\% apresentaram a forma clínica pulmonar e $28 / 33 \%$ a forma não pulmonar (Figura 1A).

$\mathrm{O}$ número de casos de pacientes positivos para tuberculose em associação com HIV/AIDS correspondeu a 32/37\% dos casos (Figura 1B).

Quanto aos hábitos dos pacientes positivos para TB, 30/34\% dos pacientes eram tabagistas, 20/23\% usuários de drogas e 14/16\% eram etilistas (Figura 1C) e os demais (36/27\%) não apresentaram hábitos prejudiciais.

Quando verificada a forma clínica dos pacientes que apresentavam a forma não pulmonar foi possível identificar que os locais acometidos com mais frequência foram: ganglionar periférica $8 / 29 \%$, intestinal $7 / 25 \%$, sistema nervoso central (SNC) $2 / 7 \%$ e óssea $2 / 4 \%$ (Tabela 2).

Pacientes acometidos em pelo menos dois locais diferentes, classificados como a forma clínica disseminada, apresentavam as seguintes localidades: ganglionar periférica e intestinal $1 / 4 \%$, ganglionar periférica e sistema nervoso central (SNC) $1 / 4 \%$ e a forma urinária atingindo os rins e bexiga 1/4\%. Em prontuários de dois pacientes não havia informação dos locais de disseminação da doença (Tabela 3).

A forma de tratamento seguiu os padrões estabelecidos pelo programa nacional de tuberculose, com $6 / 7 \%$ dos casos utilizando o esquema I (RHZ), indicado para o tratamento inicial, e $41 / 48 \%$ fizeram uso do esquema I reforçado, que associa o etambutol (E) aos demais medicamentos do esquema I. Alguns pacientes $5 / 5,8 \%$ foram tratados associando-se os dois esquemas (RHZ+RHZE) em momentos diferentes. Outros esquemas utilizando levofloxacina ou clofazimina também foram utilizados $(2 / 2 \%)$. Os demais pacientes não tiveram o esquema de tratamento relatado nos prontuários $(32 / 37,2 \%)$ (Tabela 4$)$.

Em relação ao número de óbitos, $15 / 17,4 \%$ dos pacientes faleceram durante o tratamento. Dentre esses pacientes, seis possuíam coinfecção HIV/AIDS.

Tabela 1 - Principais sinais e sintomas relatados em prontuários de pacientes com diagnóstico de tuberculose atendidos no Hospital Universitário de Londrina-PR entre os anos de 2010 e 2014.

\begin{tabular}{lcc}
\hline SINAIS E SINTOMAS & N & $\mathbf{\%}$ \\
\hline Tosse & 50 & 58 \\
Febre & 45 & 52 \\
Perda de peso & 34 & 40 \\
Sudorese noturna & 17 & 20 \\
Dispnéia & 15 & 17 \\
Náuseas e vômitos & 14 & 16 \\
Astenia & 13 & 15 \\
Diarréia & 9 & 10 \\
Anorexia & 9 & 10 \\
Cefaléia & 7 & 8 \\
Dor abdominal & 7 & 8 \\
Hemoptise & 5 & 6 \\
Linfadenomegalia & 4 & 5 \\
Hipertensão & 4 & 5 \\
Disfonia & 3 & 3 \\
Gastrite & 3 & 3 \\
Mialgia & 2 & 2 \\
Dor torácica & 2 & 2 \\
Taquicardia & 2 & 2 \\
Anemia & 2 & 2 \\
Calafrio & 1 & 1 \\
Constipação & 1 & 1 \\
Déficit motor & 1 & 1 \\
Dor de garganta & 1 & 1 \\
Asma & 1 & 1 \\
\hline To: Autos & &
\end{tabular}

Fonte: Autores. 
Figura 1 - Número de pacientes com diagnóstico de tuberculose (A), diagnóstico de tuberculose e em coinfecção com HIV/AIDS (B) e principais hábitos relatados em prontuários de pacientes com diagnóstico de tuberculose (C). Os dados representam as frequências absolutas e relativas de pacientes atendidos no Hospital Universitário de Londrina-PR, entre os anos de 2010 e 2014.
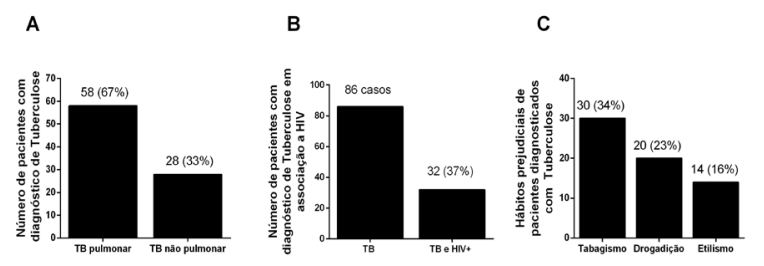

Fonte: Autores.

Tabela 2 - Formas clínicas relatadas em prontuários de pacientes com diagnóstico de tuberculose do tipo não pulmonar atendidos no Hospital Universitário de Londrina-PR entre os anos de 2010 e 2014.

\begin{tabular}{lcc}
\hline FORMA CLÍNICA NÃO PULMONAR & N & \% \\
\hline Ganglionar periférica & 8 & 29 \\
Intestinal & 7 & 25 \\
Sistema Nervoso Central (SNC) & 2 & 7 \\
Óssea & 2 & 4 \\
Articular & 1 & 4 \\
Pleural & 1 & 4 \\
Cutânea & 1 & 4 \\
Laríngea & 1 & 4 \\
\hline
\end{tabular}

Fonte: Autores.

Tabela 3 - Formas clínicas disseminadas relatadas em prontuários de pacientes com diagnóstico de tuberculose do tipo não pulmonar atendidos no Hospital Universitário de Londrina-PR entre os anos de 2010 e 2014.

\begin{tabular}{lcc}
\hline FORMA CLÍNICA DISSEMINADA & N & \% \\
\hline Ganglionar periférica + Intestinal & 1 & 4 \\
Ganglionar periférica + SNC & 1 & 4 \\
Urinária atingindo: Rins + Bexiga & 1 & 4 \\
Disseminada sem relato dos locais & 2 & 7 \\
\hline
\end{tabular}

Fonte: Autores.
Tabela 4 - Esquemas terapêuticos relatados em prontuários de pacientes com diagnóstico de tuberculose atendidos no Hospital Universitário de Londrina-PR entre os anos de 2010 e 2014.

\begin{tabular}{lcc}
\hline ESQUEMAS TERAPÊTICOS & N & $\mathbf{\%}$ \\
\hline Rifampicina, isoniazida e pirazinamida (RHZ) & 6 & 7 \\
Etambutol, rifampicina, isoniazida e pirazinamida & 41 & 48 \\
(RHZE) & 5 & 6 \\
RHZ e posteriormente RHZE & 2 & 1 \\
Levofloxacina & 2 & 1 \\
Clofazimina & 32 & 37 \\
Sem relato & & \\
\hline
\end{tabular}

Fonte: Autores.

\section{Discussão}

Dados epidemiológicos do Paraná e Londrina compreendidos entre os anos de 2010 a 2014 apontam Londrina como a segunda cidade com maior número de casos confirmados de tuberculose no estado. Neste mesmo período a região de Londrina apresentou 836 casos notificados sendo que destes 488 apresentavam confirmação laboratorial (58\%), sendo a maioria homens $(75 \%)$, com faixa etária compreendia de recém-nascidos a maiores de 80 anos e 119 casos (14\%) coinfectados com HIV/AIDS (BRASIL, 2016).

A cidade de Londrina conta com diversos níveis de atenção à saúde, inclusive com ambulatório referência para o tratamento de TB e AIDS. Sendo assim, durante o período de estudo realizado no Hospital Universitário de Londrina estavam disponíveis para a análise 86 prontuários de pacientes com diagnóstico positivo para TB.

Encontramos em nosso estudo predomínio de pacientes do sexo masculino positivos para tuberculose (76\%), o que corrobora com os dados notificados no município durante este período (BRASIL, 2016) e também com outros autores que apontam maior acometimento em pacientes do gênero masculino (CHEADE et al., 2009; LEMOS et al., 2012; SANDGREN; HOLLO; VAN DER WERF, 2013). Segundo Lemos et al. (2012) a combinação de fatores responsáveis pela diferença social entre homens e mulheres, decorrentes de distinções comportamentais e culturais podem estar relacionados a este aumento.

Dentre os sinais clínicos relatados nos prontuários analisados, destacam-se a tosse, febre, perda de peso, sudorese noturna, dispneia, hemoptise, linfadenomegalia, disfonia, dor torácica, anemia, calafrio, déficit motor, dor de garganta e asma estando 
presentes no início da infecção e são típicos de doença com acometimento pulmonar (ALARCÓN-ROBAYO et al., 2016). Por outro lado, sinais e sintomas como: náuseas e vômitos, astenia, diarreia, anorexia, cefaleia, dor abdominal, hipertensão, gastrite, mialgia, taquicardia e constipação podem ser gerados como reações adversas aos medicamentos utilizados no tratamento e, portanto, aparecendo após o inicio da terapêutica (BRASIL, 2015).

Alcântara et al. (2012) realizaram um estudo transversal entre 2008 e 2009 em três unidades de saúde de Fortaleza-CE. Os autores revelaram que anorexia, perda de peso e febre estavam associados à tuberculose pulmonar, ressaltando que a perda de peso poderia ser usada como indicador de TB pulmonar em pacientes com tosse crônica com duração acima de duas semanas.

A perda de peso foi o terceiro (40\%) sinal clínico mais apresentado pelos pacientes em nosso levantamento. Deficiências de micronutrientes, em especial, vitaminas e minerais são importantes no metabolismo, na função celular e defesa contra tuberculose (BHARGAVA et al, 2014; HAYASHI et al., 2014; KANT; GUPTA; AHLUWALIA, 2015; TANG; YAM; CHEN, 2016; WHO, 2013). O conhecimento dos efeitos de micronutrientes e suas interações com tuberculose/HIV é de grande importância nas estratégias abrangentes de planejamento para promover a saúde através da nutrição e para aumentar a eficácia da terapia específica (AMARE et al., 2015).

Nossa análise apontou que dos 86 casos de tuberculose, 28 casos foram de tuberculose não pulmonar, correspondendo a 33\%. Segundo Yasui (2014), a maioria dos casos de adultos com tuberculose secundária não pulmonar é induzida por reativação endógena, quando os bacilos se multiplicam rapidamente por falha do controle imunológico do hospedeiro. Fatores como desnutrição, envelhecimento, dosagem de medicamento imunossupressor e infecção por HIV podem estar envolvidos nessa ativação (RODRIGUES; FIEGENBAUM; MARTINS, 2010).

Dentre os casos analisados foi possível observar que pacientes que apresentavam formas clínicas do tipo não pulmonar eram acometidos principalmente nos gânglios, intestino, sistema nervoso central e ossos. Além disso, cinco pacientes foram acometidos em mais de uma região sem contar a região pulmonar e pleural, sendo estes considerados em condição de tuberculose disseminada.
Outras aspectos evidenciam que um número considerável de pacientes apresentavam hábitos nocivos como o tabagismo e alcoolismo, o que os tornam mais susceptíveis à infecção por tuberculose. O mesmo pode ser observado em estudo realizado no município de Londrina-PR, nos anos de 2001 a 2008, onde se observou que dentre os agravos comumente associados à tuberculose, AIDS e o alcoolismo foram os mais frequentes (COSTA JUNIOR, 2011).

Os dados analisados neste estudo apontaram 32 casos positivos para tuberculose/HIV com idade entre 24 e 59 anos, padrão semelhante aos casos relatados anteriormente na literatura, onde demais autores identificaram que a faixa etária predominante nos casos de tuberculose/HIV ocorre em jovens adultos, compondo uma parcela significativa da população economicamente ativa (CHEADE et al., 2009; LEMOS et al., 2012; SANDGREN; HOLLO; VAN DER WERF, 2013). No Brasil, a faixa etária dos indivíduos mais atingidos pela tuberculose compreende dos 20 aos 49 anos, abrangendo em torno de $63 \%$ dos casos novos da doença registrados em 2009. Uma das possíveis explicações para a elevada frequência da coinfecção em jovens adultos relaciona-se aos hábitos adotados por estes, uma vez que estão mais expostos ao M. tuberculosis e ao HIV (LEMOS et al., 2012; RODRIGUES; FIEGENBAUM; MARTINS, 2010).

O teste rápido para HIV é uma recomendação do Ministério da Saúde voltada para todos os pacientes com tuberculose, isso se deve ao fato de as pessoas com HIV estarem mais propensas a desenvolver a tuberculose ativa em comparação à população geral, sendo frequente o diagnóstico da infecção pelo HIV ocorrer durante o curso da tuberculose (BRASIL, 2013). Estas recomendações são principalmente porque dentre as doenças que são consideradas as principais responsáveis pela situação de calamidade pública e econômica mundial estão a tuberculose, AIDS e a malária. Más condições socioeconômicas contribuem para maior vulnerabilidade da população às doenças infectocontagiosas, principalmente pela desnutrição, a falta de acesso aos serviços de saúde e meios de prevenção. Além disso, essas doenças podem interferir no desenvolvimento econômico desses países por acometer a população economicamente ativa (DI GENNARO et al., 2017).

A imunodepressão em pacientes coinfectados com tuberculose/HIV é uma das causas mais frequentes de morte (TRINH et al., 2016). Nossos achados ( $n=6 / 40 \%)$ demonstram a grave relação entre as duas morbidades, 
indo de encontro com demais estudos, onde relataram que os pacientes com TB também tiveram uma progressão mais rápida para infecções oportunistas associadas à AIDS, sugerindo que os mecanismos para este aumento da mortalidade foi devido ao déficit da imunidade (KABALI et al., 2013). A infecção por HIV envolve mecanismos que conduzem à reativação de $M$. tuberculosis nesses pacientes. O vírus é capaz de alterar a função das células $\mathrm{T} C D 4^{+}$que coordenam a defesa imunológica contra vírus e bactérias, aumentando assim em 20 vezes a probabilidade de conversão de TB latente em tuberculose ativa, que é uma das principais causas de morte em indivíduos acometidos pela AIDS (LEMOS et al., 2012).

Com o objetivo de controlar a doença, a Organização Mundial de Saúde recomenda o tratamento completo em, no mínimo, $85 \%$ dos casos diagnosticados. Para que isso seja possível, é necessária uma rede de serviços bem organizada e gerenciada, além de informações de qualidade que são fundamentais para a implementação dessa estratégia. Neste contexto, o Hospital Universitário de Londrina é referência à nível terciário para o tratamento de tuberculose, e apresentase como um dos municípios prioritários de acordo com o Programa Nacional de Controle da Tuberculose (BRASIL, 2014).

Em estudo retrospectivo, Seiscento (2012) demonstrou que o tratamento da tuberculose em pacientes imunossuprimidos (infecção pelo HIV, diabetes e insuficiência renal) pode causar diversos efeitos colaterais como insuficiência renal e outras doenças pulmonares. No entanto, o autor ressalta a importância de um monitoramento do paciente durante o tratamento devido aos efeitos colaterais das drogas antituberculose. Visando isso, profissionais de Unidades de Atenção Básica são capacitados para o aconselhamento e a execução de testes rápidos antiHIV e de testes tuberculínicos, seguindo a orientação de descentralizar e agilizar o diagnóstico de coinfecção tuberculose/HIV e, da mesma forma, o tratamento da tuberculose latente (SEISCENTO, 2012). Entretanto, muitos pacientes não aderem ao tratamento ou abandonam após um período.

Diante das precárias condições socioeconômicas, o controle da tuberculose permanece um desafio. A desnutrição, a falta de acesso e recursos fornecidos aos serviços públicos de saúde contribui para a vulnerabilidade da população e para a prevalência de doenças infectocontagiosas. Em âmbito global, a infecção pelo HIV tem modificado a tendência epidemiológica da tuberculose, principalmente em países onde há índices elevados da doença (KWAN; ERNST, 2011; TANG; YAM; CHEN, 2016). Apesar do Brasil ter apresentado uma diminuição no coeficiente de incidência de TB, existem desafios para a redução do número de casos da doença, visto que o país ainda registra cerca de 73 mil casos novos por ano.

Sendo assim, são de grande responsabilidade a investigação e a prevenção da tuberculose, exigindo maior atenção dos profissionais e órgãos competentes, uma vez que representa um problema grave para a saúde pública. Isso porque, os aspectos decorrentes da baixa suspeição da doença e do atraso na solicitação do exame de baciloscopia de escarro, podem prejudicar o diagnóstico precoce (PONCE et al., 2016)

No intuito de se diagnosticar rapidamente, diminuindo números de pessoas infectadas e consequentemente número de óbitos, um teste inovador para a identificação rápida da tuberculose é oferecido no Sistema Único de Saúde. No exame de baciloscopia do escarro, o resultado leva 24 horas e outros 60 dias para a análise da cultura de identificação de micobactérias. O teste GeneXpert é totalmente automatizado, identificando-se fragmentos do DNA da micobactéria no escarro dentro de duas horas e com risco mínimo de contaminação (BRASIL, 2014).

Nosso estudo apresenta a importância da TB em diversos aspectos e aponta dados que permitem maiores esclarecimentos sobre o assunto, principalmente em relação ao elevado número de casos de coinfecção com HIV e pacientes com evolução à óbito em nossa região, contribuindo assim, para o desenvolvimento e implementação de campanhas ou projetos que visem o diagnóstico e tratamento precoce, proporcionando a melhoria da qualidade de vida e cura dos pacientes portadores de TB.

\section{Agradecimentos}

Coordenação de Aperfeiçoamento de Pessoal de Nível Superior (CAPES) e Universidade Estadual de Londrina (UEL).

\section{Referências}

ALARCÓN-ROBAYO, J. F.; MARTINEZ-CASALLAS, L.; SAMIR-SÁNCHEZ, M.; VALDERRAMA-MENDOZA, J. S.; Diego BADOS-ENRIQUEZ, M.; JIMÉNEZCANIZALES, C. E. Prevalencia de tuberculosis pulmonar en población privada de la libertad de 10 centros penitenciarios en Colombia, 2013. Aug. 2016. Disponível em: <https://www.researchgate.net/ 
publication/313979214_Prevalencia_de_tuberculosis_ pulmonar_en_poblacion_privada_de_la_libertad de_10_centros_penitenciarios_en_Colombia_2013 >. Accesso em: 11 set. 2016.

ALCÂNTARA, C. C. S.; KRITSKI, A. L.; FERREIRA, V. G.; FAÇANHA, M. C.; PONTES, R. S.; MOTA, R. S.; LEITÃO, T. M. J. S. Fatores associados à tuberculose pulmonar em pacientes que procuraram serviços de saúde de referência para tuberculose. Jornal Brasileiro de Pneumologia, São Paulo, v. 38, n. 5, p. 622-629, 2012.

AMARE, B.; MOGES, B.; MULU, A.; YIFRU, S.; KASSU, A. Quadruple burden of HIV/AIDS, tuberculosis, chronic intestinal parasitoses, and multiple micronutrient deficiency in ethiopia: a summary of available findings. BioMed Research International, New York, v. 2015, 2015.

ARORA, R.; ARMITIGE, L.; WANGER, A.; HUNTER, R. L.; HWANG, S. A. Association of pellicle growth morphological characteristics and clinical presentation of Mycobacterium tuberculosis isolates. Tuberculosis, Edinburgh, v. 101, p. S63-S68, 2016.

BHARgaVA, A.; BENEDETTI, A.; OXLADE, O.; PAI, M.; MENZIES, D. Undernutrition and the incidence of tuberculosis in India: national and subnational estimates of the population-attributable fraction related to undernutrition. The National Medical Journal of India, New Delhi, v. 27, n. 3, p. 128-133, 2014.

BINI, E. I.; D’ATTILIO, L.; MARQUINA-CASTILLO, B.; MATA-ESPINOSA, D.; DÍAZ, A.; MARQUEZVELASCO, R.; GAMBOA-DOMÍNGUEZ, A.; BAY, M. L.; HERNÁNDEZ-PANDO, R.; BOTTASSO, O. The implication of pro-inflammatory cytokines in the impaired production of gonadal androgens by patients with pulmonary tuberculosis. Tuberculosis, Edinburgh, v. 95 , n. 6, p. 701-706, 2015.

BOGAZ, C. Incidência da tuberculose cai 20,2\% no Brasil em uma década. Disponível em: <http://portalsaude. saude.gov.br/index.php/cidadao/principal/agenciasaude/22736-incidencia-da-tuberculose-cai-20-2-nobrasil-em-uma-decada>. Acesso em: 11 set. 2016.

BRASIL. Ministério da Saúde. Detectar, tratar e curar: desafios e estratégias brasileiras frente à tuberculose. Brasília: Ministério da Saúde, 2015.

BRASIL. Ministério da Saúde. Panorama da tuberculose no Brasil: indicadores epidemiológicos e operacionais. Brasília: Ministério da Saúde, 2014.

BRASIL. Ministério da Saúde. Recomendações para o manejo da coinfecção TB-HIV em serviços de atenção especializada a pessoas vivendo com HIV/aids. Brasília: Ministério da Saúde, 2013.
BRASIL. Ministério da Saúde. Sistema de informação de agravos de notificação (SINAN). 2016. Disponível em: $<$ http://www2.datasus.gov.br/DATASUS/index.php?are $\mathrm{a}=0203 \& \mathrm{id}=31009407 \& \mathrm{VObj}=\mathrm{http}: / /$ tabnet.datasus.gov. $\mathrm{br} / \mathrm{cgi} /$ tabcgi.exe? sinannet/cnv/tuberc $>$. Acesso em: 18 mar. 2017.

CHEADE, M. F. M.; IVO, M. L.; SIQUEIRA, P. H.; SÁ, R. G.; HONER, M. R. Characterization of tuberculosis among HIV/AIDS patients at a referral center in Mato Grosso do Sul. Revista da Sociedade Brasileira de Medicina Tropical, Uberaba, v. 42, n. 2, p. 119-125, 2009.

CLIFFORD, V.; ZUFFEREY, C.; STREET, A.; DENHOLM, J.; TEBRUEGGE, M.; CURTIS, N. Cytokines for monitoring anti-tuberculous therapy: a systematic review. Tuberculosis, Edinburgh, v. 95, n. 3, p. 217-228, 2015.

COSTA JUNIOR, W. L. Avaliação dos casos de tuberculose notificados no município de Londrina no período de 2001 a 2008. 2011. 51 f. Dissertação (Mestrado Profissional em Gestão de Serviços de Saúde) - Universidade Estadual de Londrina, Londrina, 2011.

DI GENNARO, F.; PIZZOL, D.; CEBOLA, B.; STUBBS, B.; MONNO, L.; SARACINO, A.; LUCHINI, C.; SOLMI, M.; SEGAFREDO, G.; PUTOTO, G.; VERONESE, N. Social determinants of therapy failure and multi drug resistance among people with tuberculosis: A review. Tuberculosis, Edinburgh, v. 103, p. 44-51, 2017.

EL KAMEL, A.; JOOBEUR, S.; SKHIRI, N.; MHAMED, S. C.; MRIBAH, H.; ROUATBI, N. Fight against tuberculosis in the world. Revue De Pneumologie Clinique, Paris, v. 71, n. 2-3, p. 181-187, 2015.

ETNA, M. P.; GIACOMINI, E.; SEVERA, M.; COCCIA, E. M. Pro-and anti-inflammatory cytokines in tuberculosis: a two-edged sword in TB pathogenesis. Seminars in Immunology, Philadelphia, v. 26, n. 6, p. 543-551, 2014.

GEORGE, P. J.; KUMAR, N. P.; JAGANATHAN, J.; DOLLA, C.; KUMARAN, P.; NAIR, D.; BANUREKHA, V. V.; SHEN, K.; NUTMAN, T. B.; BABU, S. Modulation of pro-and anti-inflammatory cytokines in active and latent tuberculosis by coexistent Strongyloides stercoralis infection. Tuberculosis, Edinburgh, v. 95, n. 6, p. 822-828, 2015.

HAYASHI, S.; TAKEUCHI, M.; HATSUDA, K.; OGATA, K.; KURATA, M.; NAKAYAMA, T.; OHISHI, T.; NAKAMURA, $\mathrm{H}$. The impact of nutrition and glucose intolerance on the development of tuberculosis in Japan. The International Journal of Tuberculosis and Lung Disease, Paris, v. 18, n. 1, p. 84-88, 2014. 
Perfil clínico de pacientes com diagnóstico de tuberculose atendidos no Hospital Universitário de Londrina...

HOSSAIN, M. M.; NORAZMI, M. N. Pattern recognition receptors and cytokines in Mycobacterium tuberculosis infection - the double-edge sword? BioMed Research International, New York, 2013, 2013.

KABALI, C.; MTEI, L.; BROOKS, D. R.; WADDELL, R.; BAKARI, M.; MATEE, M.; ARBEITF, R. D.; PALLANGYO, K.; HORSBURGH, C. R. Increased mortality associated with treated active tuberculosis in HIV-infected adults in Tanzania. Tuberculosis, Edinburgh, v. 93, n. 4, p. 461-466, 2013.

KANT, S.; GUPTA, H.; AHLUWALIA S. Significance of nutrition in pulmonary tuberculosis. Critical Reviews in Food Science and Nutrition, Boca Raton, v. 55, n. 7, p. 955963, 2015.

KASSA, D.; DE JAGER, W.; GEBREMICHAEL, G.; ALEMAYEHU, Y.; RAN, L.; FRANSEN, J.; WOLDAY, D.; MESSELE, T.; TEGBARU, B.; OTTENHOFF, T. H. M.; VAN BAARLE, D. The effect of HIV coinfection, HAART and TB treatment on cytokine/chemokine responses to Mycobacterium tuberculosis (Mtb) antigens in active TB patients and latently Mtb infected individuals. Tuberculosis, Edinburgh, v. 96, p. 131-140, 2016.

KWAN, C. K.; ERNST, J. D. HIV and tuberculosis: a deadly human syndemic. Clinical Microbiology Reviews, Washington, v. 24, n. 2, p. 351-376, 2011.

LAI, R. P.; NAKIWALA, J. K.; MEINTJES, G.; WILKINSON, R. J. The immunopathogenesis of the HIV tuberculosis immune reconstitution inflammatory syndrome. European Journal of Immunology, Weinheim, v. 43, n. 8, p. 1995-2002, 2013.

LEMOS, L. A.; FEIJÃO, A.R.; GIR, E.; GALVÃO, M. T. G. Aspectos da qualidade de vida de pacientes com coinfecção HIV/tuberculose. Acta Paulista de Enfermagem, São Paulo, v. 25, n. 1, p. 41-47, 2012.

O'GARRA, A.; REDFORD, P. S.; MCNAB, F. W.; BLOOM, C. I.; WILKINSON, R. J.; BERRY, M. P. The immune response in tuberculosis. Annual Review of Immunology, Palo Alto, v. 31, p. 475-527, 2013.

PONCE, M. A. Z.; WYSOCKI, A. D.; ARAKAWA, T.; ANDRADE, R. P.; VENDRAMINI, S. H. F.; SILVA SOBRINHO, R. A.; MONROE, A. A.; RUFFINO NETTO, A.; VILLA, T. C. S. Atraso do diagnóstico da tuberculose em adultos em um município paulista em 2009: estudo transversal. Epidemiologia e Serviços de Saúde, Brasília, v. 25, n. 3, p. 553-562, 2016.

RODRIGUES, J. L. C.; FIEGENBAUM, M.; MARTINS, A. F. Prevalence of tubeculosis/HIV coinfection in patients from Model Health Centre in Porto Alegre, Rio Grande do Sul. Scientia Medica, Porto Alegre, v. 20, n. 3, p. 212217, 2010.
SANDGREN, A.; HOLLO, V.; VAN DER WERF, M. J. Extrapulmonary tuberculosis in the European Union and European Economic Area, 2002 to 2011. Euro Surveillance, Sweden, v. 18, n. 12, p. 1-9, 2013.

SEISCENTO, M. Tuberculosis in special settings: HIV, diabetes mellitus and renal failure. Pulmão, Rio de Janeiro, v. 21, n. 1, p. 23-26, 2012.

SHETE, P. B.; HAGUMA, P.; MILLER, C. R.; OCHOM, E.; AYAKAKA, I.; DAVIS, J. L.; DOWDY, D. W.; HOPEWELL, P.; KATAMBA, A.; CATTAMANCHI, A. Pathways and costs of care for patients with tuberculosis symptoms in rural Uganda. The International Journal of Tuberculosis and Lung Disease, Paris, v. 19, n. 8, p. 912-917, 2015.

TANG, J.; YAM, W. C.; CHEN, Z. Mycobacterium tuberculosis infection and vaccine development. Tuberculosis, Edinburgh, v. 98, p. 30-41, 2016.

TRINH, Q. M.; NGUYEN, H. L.; DO, T. N.; NGUYEN, V. N.; NGUYEN, B. H.; NGUYEN, T. V.; SINTCHENKO, V.; MARAIS, B. J. Tuberculosis and HIV co-infection in Vietnam. International Journal of Infectious Diseases, Brookline, v. 46, p. 56-60, 2016.

WHO - WORLD HEALTH ORGANIZATION. Global tuberculosis report 2015. Geneva, 2015.

WHO - WORLD HEALTH ORGANIZATION. Tuberculosis. Saudi Medical Journal, Riyadh, v. 34, n. 11, p. 1205e7, 2013.

WU, J.; WANG, S.; LU, C.; SHAO, L.; GAO, Y.; ZHOU, Z.; HUANG, H.; ZHANG, Y.; ZHANG, W. Multiple cytokine responses in discriminating between active tuberculosis and latent tuberculosis infection. Tuberculosis, Edinburgh, v. 102, p. 68-75, 2017.

YASUI, K. Immunity against Mycobacterium tuberculosis and the risk of biologic anti-TNF- $\alpha$ reagents. Pediatric Rheumatology, Brussels, v. 12, n. 1, p. 45, 2014.

Recebido em: 06 out. 2016

Aceito em: 06 mar. 2017 
Bosqui, L. R. et al. 